\title{
A GENERALIZATION OF A THEOREM OF S. N. BERNSTEIN ${ }^{1}$
}

\author{
J. D. CHANDLER, JR.
}

\begin{abstract}
A rational approximation scheme is exhibited for a set $\Delta$ which consists of a finite union of compact subintervals of the real line. This rational approximation scheme provides a characterization of the analytic functions on $\Delta$ which generalizes $S$. N. Bernstein's characterization of the analytic functions on $[-1,1]$.
\end{abstract}

1. Bernstein's theorem. Let $E$ be a compact subset of the real line $R$. A complex-valued function $f$ defined on $E$ is said to be analytic on $E$ if $f$ is the restriction to $E$ of a holomorphic function $F$ defined on an open subset $\Omega$ of the complex plane $\mathbf{C}$ that contains $E$. Note that $\Omega$ need not be a region.

A theorem of S. N. Bernstein relates the analyticity of a function $f$ defined on the interval $I=[-1,1]$ to the rate by which $f$ may be uniformly approximated by polynomials on $I$. In order to state Bernstein's theorem precisely, let $P_{n}$ be the class of all polynomials of degree $\leqslant n$, let

$$
\|g\|_{I}=\sup \{|g(x)|: x \in I\}
$$

for any function $g$ defined on $I$, and let

$$
e\left(f, P_{n}, I\right)=\inf \left\{\|f-p\|_{I}: p \in P_{n}\right\} \quad(n=1,2,3, \ldots) .
$$

Let $D_{\rho}$ be the open elliptical region defined by

$$
D_{\rho}=\{z \in \mathbf{C}:|z+1|+|z-1|<\rho+1 / \rho\},
$$

for $0<\rho<1$.

THEOREM 1 (S. N. BERNSTEIN). Let $f$ be a complex-valued function defined on $I=[-1,1]$. Then

$$
\limsup _{n \rightarrow \infty} \sqrt[n]{e\left(f, P_{n}, I\right)} \leqslant \rho
$$

with $0<\rho<1$, if and only if $f$ is the restriction to I of a holomorphic function $F$ defined on the region $D_{\rho}$. In particular,

$$
\lim _{n \rightarrow \infty} \sqrt[n]{e\left(f, P_{n}, I\right)}=0
$$

if and only if the holomorphic function $F$ is entire.

Proof. See [1, pp. 110-113].

Received by the editors August 20, 1976.

AMS (MOS) subject classifications (1970). Primary 41A20; Secondary 26A81, 26A90.

Key words and phrases. Cayley inner function.

${ }^{1}$ Research supported by NSF Grant GP-31483X2. 
Suppose one replaces the interval $I$ by a union of intervals

$$
\Delta=\bigcup_{j=0}^{r}\left[a_{j}, b_{j}\right]
$$

where $-\infty<a_{0}<b_{0}<a_{1}<\cdots<b_{r}<+\infty$, and seeks a similar characterization of the analytic functions on $\Delta$. If one thinks of polynomials as rational functions with poles at the point $\lambda_{0}=\infty$, then one might try to generalize Bernstein's theorem to $\Delta$ by replacing the class of polynomials by a class of rational functions with poles at certain points $\lambda_{1}, \ldots, \lambda_{r}$ between each of the intervals $\left[a_{0}, b_{0}\right],\left[a_{1}, b_{1}\right], \ldots,\left[a_{r}, b_{r}\right]$, as well as at the point $\lambda_{0}=\infty$. The difficulty in finding such a generalization is choosing the poles $\lambda_{1}, \ldots, \lambda_{r}$.

In this note the work of Rosenblum and Rovnyak on Cayley inner functions (see [3]) is used to develop a rational approximation scheme for $\Delta$ that corresponds to polynomial approximation on $I$ and provides a characterization of the analytic functions on $\Delta$ analogous to that in Bernstein's theorem.

2. Let $\Delta=\cup_{j=0}^{r}\left[a_{j}, b_{j}\right]$, where $-\infty<a_{0}<b_{0}<a_{1}<\cdots<b_{r}<+\infty$, and let $\phi$ be the rational function

$$
\phi(z)=\left(b-a \prod_{j=0}^{r} \frac{b_{j}-z}{a_{j}-z}\right) /\left(1-\prod_{j=0}^{r} \frac{b_{j}-z}{a_{j}-z}\right) .
$$

The poles of $\phi(z)$ are the solutions of the equation $1=\prod_{j=0}^{r}\left(b_{j}-z\right) /\left(a_{j}-z\right)$, and hence are at the points $z=\infty$ and $z=\lambda_{1}, \ldots, \lambda_{r}$, where $b_{j-1}<\lambda_{j}<a_{j}$ $(j=1, \ldots, r)$. Moreover,

$$
\phi^{-1}([a, b])=\Delta \text { and } \phi^{-1}\left([a, b]^{c}\right)=\Delta^{c} \backslash\left\{\lambda_{1}, \ldots, \lambda_{r}\right\}
$$

(where $E^{c}=\{x \in R: x \notin E\}$ whenever $E \subseteq R$ ), and $\phi$ is a strictly increasing mapping of each of the intervals $\left[a_{0}, b_{0}\right],\left[a_{1}, b_{1}\right], \ldots,\left[a_{r}, b_{r}\right]$ onto $[a, b]$. The rational function $\phi$ is an example of a Cayley inner function and is said to adapt $\Delta$ to $[a, b]$; see [3] for details.

Let $\lambda_{0}=-\infty, \lambda_{r+1}=+\infty$, and put

$$
\phi_{j}=\phi \mid\left(\lambda_{j}, \lambda_{j+1}\right) \quad(j=0,1, \ldots, r) .
$$

Define the functions $k_{0}, k_{1}, \ldots, k_{r}$ on $\Delta$ by

$$
k_{0}(t)=1, \quad k_{1}(t)=\left(t-\lambda_{1}\right)^{-1}, \ldots, k_{r}(t)=\left(t-\lambda_{r}\right)^{-1} .
$$

(We use $t$ as a dummy variable for functions defined on $\Delta$ and $x$ for functions defined on $[a, b]$.) If $E$ is a compact subset of the real line $R$, then $C(E)$ is the Banach space of all complex-valued continuous functions on $E$ with norm $\|f\|_{E}=\sup \{|f(x)|: x \in E\} ; C(E)^{r+1}$ is the direct sum of $r+1$ copies of $C(E)$ and is a Banach space under any of several equivalent norms.

Lemma. Define the linear operator $B: C([a, b])^{r+1} \rightarrow C(\Delta)$ by 


$$
(B g)(t)=\sum_{j=0}^{r} k_{j}(t) g_{j}(\phi(t))
$$

where $g=\left(g_{0}, g_{1}, \ldots, g_{r}\right)$. Then $B$ is bounded and has a bounded inverse defined by

$$
\left(B^{-1} f\right)(x)=A(x)^{-1} \cdot\left[\begin{array}{c}
f\left(\phi_{0}^{-1}(x)\right) \\
\vdots \\
f\left(\phi_{r}^{-1}(x)\right)
\end{array}\right], \quad(f \in C(\Delta)),
$$

where $A(x)$ is the matrix

$$
A(x)=\left[a_{i j}(x)\right]_{i, j=0}^{r}=\left[k_{j}\left(\phi_{i}^{-1}(x)\right)\right]_{i, j=0}^{r} .
$$

Proof. If $g=\left(g_{0}, g_{1}, \ldots, g_{r}\right) \in C([a, b])^{r+1}$, then

$$
\|B g\|_{\Delta} \leqslant(r+1) \cdot K \cdot \max _{j}\left\|g_{j} \circ \phi\right\|_{\Delta}=(r+1) \cdot K \cdot \max _{j}\left\|g_{j}\right\|_{[a, b]},
$$

where $K=\max _{j}\left\|k_{j}\right\|_{\Delta}<+\infty$. Hence $B$ is a bounded operator.

If $f \in C(\Delta)$, then $f=B g$ with $g=\left(g_{0}, g_{1}, \ldots, g_{r}\right)$ if and only if

$$
f\left(\phi_{i}^{-1}(x)\right)=\sum_{j=0}^{r} k_{j}\left(\phi_{i}^{-1}(x)\right) g_{j}(x) \quad(i=0,1, \ldots, r)
$$

that is, if and only if

$$
f\left(\phi_{i}^{-1}(x)\right)=\sum_{j=0}^{r} a_{i j}(x) g_{j}(x) \quad(i=0,1, \ldots, r),
$$

where $a_{i j}(x)=k_{j}\left(\phi_{i}^{-1}(x)\right)$ for $i, j=0,1, \ldots, r$. The matrix $A(x)=\left[a_{i j}(x)\right]$ has the form

$$
\left[\begin{array}{cccc}
1, & \frac{1}{\eta_{0}-\lambda_{1}} & , \ldots, & \frac{1}{\eta_{0}-\lambda_{r}} \\
\ldots & \frac{1}{\eta_{r}-\lambda_{1}} & , \ldots, & \frac{1}{\eta_{r}-\lambda_{r}}
\end{array}\right]
$$

where $\eta_{i}=\phi_{i}^{-1}(x)$. Since

$$
\operatorname{det}\left[\frac{1}{\eta_{i}-\lambda_{j}}\right]_{i, j=0}^{r}=\frac{\Pi_{r \geqslant i>j \geqslant 0}\left(\eta_{i}-\eta_{j}\right)\left(\lambda_{j}-\lambda_{i}\right)}{\prod_{i, j=0, \ldots, n}\left(\eta_{i}-\lambda_{j}\right)}
$$

(see [2, part seven, Problem 3]), we deduce that

$$
\operatorname{det} A(x)=\lim _{\lambda_{0} \rightarrow \infty}\left(-\lambda_{0} \operatorname{det}\left[\frac{1}{\eta_{i}-\lambda_{j}}\right]_{i, j=0}^{r}\right) \neq 0
$$

for $a \leqslant x \leqslant b$. Thus $A(x)$ is invertible for $a \leqslant x \leqslant b$, and 


$$
A(x)^{-1}=\left[d_{i j}(x)\right], \quad d_{i j}(x)=C_{j i}(x) / \operatorname{det} A(x),
$$

where $C_{i j}(x)$ is the cofactor of $a_{i j}(x)$ in $A(x)$. It follows that if $f$ is in $C(\Delta)$ and if

$$
g(x)=\left[\begin{array}{c}
g_{0}(x) \\
g_{1}(x) \\
\vdots \\
g_{r}(x)
\end{array}\right]=A(x)^{-1} \cdot\left[\begin{array}{c}
f\left(\phi_{0}^{-1}(x)\right) \\
f\left(\phi_{1}^{-1}(x)\right) \\
\vdots \\
f\left(\phi_{r}^{-1}(x)\right)
\end{array}\right],
$$

then $g \in C([a, b])^{r+1}, f=B g$, and $\left\|g_{i}\right\|_{[a, b]} \leqslant M\|f\|_{\Delta}(i=0,1, \ldots, r)$ for some finite constant $M$ independent of $f$. This completes the proof.

Since the matrix $A(x)=\left[a_{i j}(x)\right]$ has analytic entries, it follows that, if $f=B g$ and $g=\left(g_{0}, g_{1}, \ldots, g_{r}\right)$, then $f$ is analytic on $\Delta$ if and only if $g_{0}$, $g_{1}, \ldots, g_{r}$ are analytic on $[a, b]$. For $n=1,2,3, \ldots$, let $K_{n}$ be the complex vector space spanned by the functions

$$
1, t, \ldots, t^{n} ; \frac{1}{t-\lambda_{1}}, \ldots, \frac{1}{\left(t-\lambda_{1}\right)^{n+1}} ; \cdots ; \frac{1}{t-\lambda_{r}}, \ldots, \frac{1}{\left(t-\lambda_{r}\right)^{n+1}} \text {. }
$$

Then $K_{n}$ is the image under $B$ of all $(r+1)$-tuples of polynomials of degree $\leqslant n$; see [3, Lemma 2.4]. The class $K_{n}$ will replace the class of polynomials $P_{n}$ in our generalization of Bernstein's theorem to $\Delta$.

3. A generalization of Bernstein's theorem. For simplicity, we let $[a, b]$ be the interval $I=[-1,1]$. For each complex-valued function $f$ defined on $\Delta$, put

$$
e\left(f, K_{n}, \Delta\right)=\inf \left\{\|f-k\|_{\Delta}: k \in K_{n}\right\} \quad \text { for } n=1,2,3, \ldots
$$

THEOREM 2. A complex-valued function $f$ defined on $\Delta$ is analytic on $\Delta$ if and only if

$$
\limsup _{n \rightarrow \infty} \sqrt[n]{e\left(f, K_{n}, \Delta\right)}<1
$$

and

$$
\lim _{n \rightarrow \infty} \sqrt[n]{e\left(f, K_{n}, \Delta\right)}=0
$$

if and only if $f$ has a holomorphic extension $F$ to the region $\Omega_{\phi}=\{z \in \mathbf{C}$ : $\left.z \neq \lambda_{1}, \ldots, z \neq \lambda_{r}\right\}$. There exists a real number $\rho$ that depends only on $\phi$, with $0<\rho<1$, such that $f$ has a holomorphic extension $F$ to a region if

$$
\limsup _{n \rightarrow \infty} \sqrt[n]{e\left(f, K_{n}, \Delta\right)}<\rho
$$

Proof. Let $f$ be a function on $\Delta$ with 


$$
\limsup _{n \rightarrow \infty} \sqrt[n]{e\left(f, K_{n}, \Delta\right)}<\sigma<1
$$

Then there exists an integer $N$ such that $e\left(f, K_{n}, \Delta\right)<\sigma^{n}$ if $n \geqslant N$. Hence $f$ is the uniform limit of continuous functions on $\Delta$ and so $f \in C(\Delta)$. By the Lemma, there exist continuous functions $g_{0}, g_{1}, \ldots, g_{r}$ on $I$ such that $f=B g$, where $g=\left(g_{0}, g_{1}, \ldots, g_{r}\right)$. For each $n \geqslant N$, there is a function $p_{n}$ in $K_{n}$ with $\left\|f-p_{n}\right\|_{\Delta}<\sigma^{n}$ and there exist polynomials $p_{n 0}, p_{n 1}, \ldots, p_{n r}$ such that $p_{n}=$ $B\left(p_{n 0}, p_{n 1}, \ldots, p_{n r}\right)$. By the Lemma, there is a finite constant $M$ such that

$$
\left\|g_{j}-p_{n j}\right\|_{I} \leqslant M\left\|f-p_{n}\right\|_{\Delta}<M \sigma^{n} \quad(n \geqslant N ; j=0,1, \ldots, r) .
$$

Thus

$$
\limsup _{n \rightarrow \infty} \sqrt[n]{e\left(g_{j}, P_{n}, I\right)} \leqslant \sigma<1 \quad(j=0,1, \ldots, r)
$$

and so the functions $g_{0}, g_{1}, \ldots, g_{r}$ are analytic on $I$ by Bernstein's theorem. Thus $f=B g$ is analytic on $\Delta$.

Moreover, if (5) holds for $f$, then

$$
\limsup _{n \rightarrow \infty} \sqrt[n]{e\left(g_{j}, P_{n}, I\right)}=0 \quad(j=0,1, \ldots, r)
$$

and so each $g_{j}$ has a holomorphic extension to an entire function $G_{j}$ by Bernstein's theorem. Hence

$$
F(z)=\sum_{j=0}^{r} k_{j}(z) G_{j}(\phi(z)) \quad\left(z \in \Omega_{\phi}\right)
$$

is a holomorphic extension of $f$ to $\Omega_{\phi}$.

A simple connectedness argument shows there exists a real number $\rho$ in $(0$, 1) such that $\phi^{-1}\left(D_{\rho}\right)$ is a region, where $D_{\rho}$ is defined by (1). Suppose (6) holds for $f$. Put $\sigma=\rho$ in the above argument; then Bernstein's theorem implies that each $g_{j}$ has a holomorphic extension $G_{j}$ to $D_{\rho}$. If $F(z)=\sum_{j} k_{j}(z) G_{j}(\phi(z))$ for $z$ in $\phi^{-1}\left(D_{\rho}\right)$, then $F$ is a holomorphic extension of $f$ to the region $\phi^{-1}\left(D_{\rho}\right)$.

Conversely, if $f$ is analytic on $\Delta$, then there exist analytic functions $g_{0}$, $g_{1}, \ldots, g_{r}$ on $I$ such that $f=B g$, where $g=\left(g_{0}, g_{1}, \ldots, g_{r}\right)$. By Bernstein's theorem (2) and the Lemma, inequality (4) holds for $f$.

Suppose, in addition, that $f$ has a holomorphic extension $F$ to $\Omega_{\phi}$. Cauchy's integral formula shows that

$$
F=F_{0}+F_{1}+\cdots+F_{r},
$$

where $F_{0}$ is entire and $F_{j}$ is holomorphic in $\mathbf{C}_{\infty} \backslash\left\{\lambda_{j}\right\}$ (where $\mathbf{C}_{\infty}$ is the Riemann sphere $\mathbf{C} \cup\{\infty\}$ ), for $j=1, \ldots, r$. By Bernstein's theorem (3),

$$
\lim _{n \rightarrow \infty} \sqrt[n]{e\left(F_{0}, P_{n},\left[a_{0}, b_{r}\right]\right)}=0 .
$$

Since $P_{n} \subseteq K_{n}$ and $\Delta \subseteq\left[a_{0}, b_{r}\right]$,

$$
\lim _{n \rightarrow \infty} \sqrt[n]{e\left(F_{0}, K_{n}, \Delta\right)}=0
$$


To establish the same result for $F_{j}$, we put $\zeta_{j}(z)=\left(z-\lambda_{j}\right)^{-1}$. Then $\zeta_{j}$ maps $\mathbf{C}_{\infty} \backslash\left\{\lambda_{j}\right\}$ conformally onto $\mathbf{C}$ and maps $\left[-\infty, b_{j-1}\right] \cup\left[a_{j},+\infty\right]$ onto a compact interval $I_{j}$ of the real line. Also, $F_{j}=G_{j} \circ \zeta_{j}$, where $G_{j}$ is entire. By Bernstein's theorem (3),

$$
\lim _{n \rightarrow \infty} \sqrt[n]{e\left(G_{j}, P_{n}, I_{j}\right)}=0 .
$$

Let $P_{n}\left(\lambda_{j}\right)=\left\{p \circ \zeta_{j}: p \in P_{n}\right\}$ for $n=1,2,3, \ldots ;$ then $P_{n}\left(\lambda_{j}\right) \subseteq K_{n}$. Since

$$
\left\|G_{j}-p\right\|_{I_{j}} \geqslant\left\|G_{j} \circ \zeta_{j}-p \circ \zeta_{j}\right\|_{\Delta}=\left\|F_{j}-p \circ \zeta_{j}\right\|_{\Delta} \quad\left(p \in \bigcup_{n=1}^{\infty} P_{n}\right),
$$

equation (9) implies that

$$
\lim _{n \rightarrow \infty} \sqrt[n]{e\left(F_{j}, P_{n}\left(\lambda_{j}\right), \Delta\right)}=0
$$

and hence that

$$
\lim _{n \rightarrow \infty} \sqrt[n]{e\left(F_{j}, K_{n}, \Delta\right)}=0 \quad(j=1, \ldots, r) .
$$

By (7), (8) and (10), equation (5) holds.

This completes the proof.

\section{REFERENCES}

1. S. N. Bernstein, Leçons sur les propriétés extrémales et la meilleure approximation des fonctions analytiques d'une variable réelle, Gauthiers-Villars, Paris, 1926.

2. G. Pólya and G. Szegö, Problems and theorems in analysis. Vol. II, Springer-Verlag, Berlin and New York, 1976.

3. M. Rosenblum and J. Rovnyak, Cayley inner functions and best approximations, J. Approximation Theory 17 (1976), 241-253.

Department of Mathematics, University of Virginia, Charlottesville, Virginia 22903

Current address: Department of Mathematics, University of Tennessee, Knoxville, Tennessee 37916 